\section{Downsides to the nitrate-nitrite- nitric oxide pathway in physiology and therapeutics? Reply from Lundberg, Weitzberg and Gladwin}

\section{Jon O. Lundberg, Eddie Weitzberg and Mark T. Gladwin}

We appreciate the comments relating to our Review article (The nitrate-nitrite-nitric oxide pathway in physiology and therapeutics. Nature Rev. Drug Discov. 7, 156-167 (2008)) ${ }^{1}$ from Nirmal Singh Panesar (Downsides to the nitrate-nitrite-nitric oxide pathway in physiology and therapeutics? Nature Rev. Drug Discov. 1 Aug 2008 (doi:10.1038/nrd2466-c1))2. As stated in our Review, hypoxia is certainly not a prerequisite for nitrite reduction to nitric oxide (NO); this process occurs along the physiological deoxygenation gradient ${ }^{1}$. In fact, in our own previous studies we noted vasodilation under normoxic conditions in aortic rings ${ }^{3}$ and in nitrite infusion studies in humans, during which there is physiological deoxygenation across tissue beds ${ }^{4,5}$. In addition, in studies using dietary nitrate we have observed a reduction in blood pressure in normoxic individuals ${ }^{6}$. Although we fully agree with Panesar that nitrite is bioactivated in vivo along the physiological oxygen gradient, it is clear from both in vitro studies of the enzymology of the haemoglobins ${ }^{7}$ and xanthine oxidoreductase ${ }^{8}$, and in vivo studies in animals ${ }^{9,10}$ and humans $s^{4,11}$ that nitrite-dependent signalling is potentiated as oxygen tensions decrease. This provides for graded NO production during physiological and pathological hypoxia. Mixed venous oxygen tensions represent a 'step up' in oxygen from specific organ oxygen tensions, secondary to mixing of all systemic blood flow. For example, the coronary sinus $\mathrm{PO}_{2}$ is only 19 $\mathrm{mm} \mathrm{Hg}$ at rest (haemoglobin oxygen saturation of less than $50 \%$ ), and similar oxygen extraction occurs across skeletal muscle, kidney and brain. Thus, the in vivo vasodilatory effects of nitrite occur in a system that efficiently extracts oxygen across resistance vessels at rest ${ }^{12}$.

With regard to NO and angina pectoris, the endogenous mechanisms for vasodilation are not sufficient when vascular occlusion is severe, and, in such cases, pharmacological treatment with organic nitrates, for example, may be necessary. It would be interesting to study whether therapeutic increases in circulating nitrite levels, by dietary or pharmacological interventions, could help to delay the onset of angina. We disagree about the controversy in circulating nitrite levels. Indeed, in early studies, some very high levels have been reported, but they most probably reflect the sum of nitrate and nitrite rather than only nitrite. Numerous more recent studies using different highly sensitive techniques have clearly showed that normal plasma nitrite levels in humans are in the range 50-500 nM and very seldom above $1 \mu \mathrm{M}^{1}$.

The possible interaction between the nitrate-nitrite-NO pathway and steroid hormones is interesting and clearly merits further study. Apart from the studies cited by Panesar, such interaction is also supported by a very recent study in which nitrate and nitrite were shown to potently activate oestrogen receptor- $\alpha^{13}$. In addition, nitrate may also affect the thyroid gland by interfering with iodine transport ${ }^{14}$. However, the significance of these possible negative interactions with endocrine functions remains to be established, and the numerous epidemiological studies in humans performed over the past 40 years do not support any major negative health effects of a diet that is high in nitrate ${ }^{14}$.
Jon O. Lundberg is at the Department of Physiology and Pharmacology, Karolinska Institute, SE-171 77 Stockholm, Sweden.

Eddie Weitzberg is at the Section of Anesthesiology and Intensive Care, Department of Physiology and Pharmacology, Karolinska Institute, SE-171 77 Stockholm

Mark T. Gladwin is at the Pulmonary and Vascular Medicine Branch, National Heart, Lung, and Blood Institute, National Institutes of Heath, Bethesda, Maryland 20892, USA, and at the Critical Care Medicine Department, Clinical Center, National Institutes of Health, Bethesda.

e-mails: jon.lundberg@ki.se; eddie.weitzberg@ki.se; mgladwin@mail.nih.gov

Lundberg, J. O., Weitzberg, E. \& Gladwin, M. T. The nitrate-nitrite-nitric oxide pathway in physiology and therapeutics. Nature Rev. Drug Discov. 7, 156-167 (2008).

2. Panesar, N. S. Downsides to the nitrate-nitrite-nitric oxide pathway in physiology and therapeutics? Nature Rev. Drug Discov. 1 Aug 2008 (doi:10.1038/ nrd2466-c1).

3. Modin, A. et al. Nitrite-derived nitric oxide: a possible mediator of 'acidic-metabolic' vasodilation. Acta Physiol. Scand. 171, 9-16 (2001).

4. Cosby, K. et al. Nitrite reduction to nitric oxide by deoxyhemoglobin vasodilates the human circulation. Nature Med. 9, 1498-1505 (2003).

5. Dejam, A. et al. Nitrite infusion in humans and nonhuman primates: endocrine effects, pharmacokinetics, and tolerance formation. Circulation 116, 1821-1831 (2007).

6. Larsen, F. J., Ekblom, B., Sahlin, K., Lundberg, J. O. $\&$ Weitzberg, E. Effects of dietary nitrate on blood pressure in healthy volunteers. N. Engl. J. Med. 355, 2792-2793 (2006).

7. Huang, Z. et al. Enzymatic function of hemoglobin as a nitrite reductase that produces NO under allosteric control. J. Clin. Invest. 115, 2099-2107 (2005).

8. Millar, T. M. et al. Xanthine oxidoreductase catalyses the reduction of nitrates and nitrite to nitric oxide under hypoxic conditions. FEBS Lett. 427, 225-228 (1998).

9. Jansson, E. A. et al. A mammalian functional nitrate reductase that regulates nitrite and nitric oxide homeostasis. Nature Chem. Biol. 4, 411-417 (2008)

10. Duranski, M. R. et al. Cytoprotective effects of nitrite during in vivo ischemia-reperfusion of the heart and liver. J. Clin. Invest. 115, 1232-1240 (2005).

11. Maher, A. R. et al. Hypoxic modulation of exogenous nitrite-induced vasodilation in humans. Circulation 117, 670-677 (2008).

12. Tsai, A. G., Johnson, P. C. \& Intaglietta, M. Oxygen gradients in the microcirculation. Physiol. Rev. 83, 933-963 (2003)

13. Veselik, D. J. et al. Activation of estrogen receptor- $\alpha$ by the anion nitrite. Cancer Res. 68, 3950-3958 (2008).

14. Speijers, G. J. A. \& van den Brandt, P. A. in Safety Evaluation of Certain Food Additives and Contaminants. Fifty-ninth meeting of the Joint FAO WHO Expert Committee on Food Additives (JECFA). WHO Food Additives Series No 50. (World Health Organization, Geneva, 2003) 